\title{
Multi-scale modelling of $E$. coli metabolism
}

\author{
Kieran Smallbone $e^{1,2, *}$ \\ Pierre Millard ${ }^{3,4,5}$ \\ ${ }^{1}$ Manchester Centre for Integrative Systems Biology \\ ${ }^{2}$ School of Computer Science \\ University of Manchester, UK. \\ ${ }^{3}$ Université de Toulouse; INSA, UPS, INP, LISBP \\ ${ }^{4}$ INRA, UMR792 Ingénierie des Systémes Biologiques et des Procédés \\ ${ }^{5}$ CNRS, UMR5504 \\ Toulouse, France.
}

\begin{abstract}
In response to environmental and genetic perturbations, micro-organisms may regulate their metabolism both metabolically - via metabolite-enzyme interactions - and hierarchically - via modulating enzyme capacities. In this report, we develop a combined metabolic and genetic regulatory model of $E$. coli that may be used to test this multiscale response.
\end{abstract}

\footnotetext{
${ }^{*}$ To whom correspondence should be addressed at kieran.smallbone@manchester.ac.uk
} 


\section{Metabolic module}

A number of kinetic models of $E$. coli metabolism are available in the literature [1, 2, 3, 4, 5, varying both in their level of detail and in their availability in standardised formats. As a scaffold, we use the metabolic model of Chassagnole et al. [1], available from the BioModels database [6] in SBML format [7] with accession number BIOMD0000000051, Some changes are made to this model, to enhance its use:

- co-metabolites $\mathrm{AxP}$ and $\mathrm{NAD} / \mathrm{P} / \mathrm{H}$ are allowed to vary

- dilution of intracellular pools due to growth, which have a negligible effect on system dynamics, are removed

- glucose uptake is set to an experimentally-determined initial rate of $1.23 \mathrm{mM} \mathrm{s}^{-1}$, of which $22 \%$ enters the pentose phosphate pathway [8]

- rMax values are rescaled to ensure the system starts at steady state, as was done in the original paper

- biomass-producing branches (that is, those other than GPS, PDH and PPC) are coupled via a single reaction, to simulate growth at an initial rate of $1.67 \times 10^{-4} \mathrm{~s}^{-1}$. This ensures that all the metabolic building blocks are used in stoichiometric proportions dictated by the cell composition, and links metabolism to overall cell physiology.

\section{Integration of metabolism with regulation of gene expression}

The model of Nishio et al. [5] contains a detailed description of the glucose phosphotransferase system (PTS) - the mechanism via which E. coli uptakes glucose - and its genetic regulation. We encoded their model in SBML format, now available from the BioModels database with accession number MODEL1501300000.

We found that the Nishio model shows a non-monontonic response of glucose flux to changes in glucose levels: as glucose concentration increases beyond approximately $0.01 \mathrm{mM}$, glucose uptake rate decreases. This non-physiological response appears to be due to an imbalance between the "accelerator" and "brake" modules. Since this balance is controlled by the "computer" module, we focused on this module to rebalance the two others. By increasing the affinity of CYA for IIAP - changing the parameter $\mathrm{kb}$ in reaction binding_IIA_P_Cya from 100 to $5000 \mathrm{mM}^{-2}$ - this non-physiological behaviour is alleviated.

We rescale glucose flux, as above, to rate $1.23 \mathrm{mMs}^{-1}$. This means that Nishio, and the metabolic module set out above, are compatible models: their overlapping elements - the PTS system - have equal fluxes, and the two models may be easily merged by removing the simple PTS reaction from the metabolic model, and appending the entire Nishio model. 


\section{Expansion of the regulatory module}

The model is then expanded through inclusion of transcription and translation for all the metabolic enzymes. This is achieved by using the transcriptional and translational reactions of Nishio to guide us as to typical parameter values for these processes. The approach of building models using typical parameter values has been used previously to derive large-scale metabolic models [9, 10]; the justification is that model is largely driven by its structure, and hence approximate parameter values are often sufficient to produce correct overall behaviour. Typical parameter values are set out in Table 1.

We finally added transcriptional regulation, in addition to that already in present in Nishio. Included regulatory interactions are set out in Table 2 ,

\section{Evaluation of predictive capabilities}

The model developed here simulates the steady-state metabolic operation of E. coli growing under abundant glucose. To test the predictive capabilities of this model, we simulate both the time-course response of $E$. coli to a sudden decrease of glucose level (from $2 \mathrm{mM}$ to $10 \mu \mathrm{M}$, Figure 1), and the steady-state reached by the system under a large range of glucose levels (from $10 \mu \mathrm{M}$ to $10 \mathrm{mM}$, Figure 2).

Firstly, the metabolic and regulatory modules show different dynamics in response to a sudden decrease of glucose concentration. While the metabolic module responds rapidly (in the second to minute range), the regulatory module shows a slower response (in the minute to hour range), as expected.

Secondly, both the steady-state glucose uptake and growth rates monotonically increase with the glucose level. This is qualitatively consistent with observations, although the predicted growth rate is higher than measured at low glucose levels. Expanding the model with additional metabolic pathways and/or regulatory interactions, as well as performing a new round of calibration, may improve these predictions. Regarding intracellular fluxes, the partition of carbon at the glycolysis-pentose phosphate pathway node is stable for all glucose levels, which is in excellent agreement with experimental data [11.

\section{Conclusion}

We present a combined metabolic and genetic regulatory model of E. coli. Simulation results indicate that, while the model is developed and calibrated using experimental data collected under a unique metabolic steady-state, the predicted flux responses to perturbations are consistent with current knowledge. Its predictive capabilities will be improved by refining parameters and expanding it with additional metabolic and regulatory processes (e.g. posttranscriptional regulation).

This model may be useful in the field of systems biology, to investigate the specific roles of metabolic and hierarchical regulation in the long-term response of E. coli to environmental 
78 and genetic perturbations. It may also assist the design of more efficient and robust cell 79 factories in biotechnology.

80 The model outlined here is available from the BioModels database with accession number 81 MODEL1503050000.

82 Acknowledgements KS was funded by the EU FP7 (KBBE) grant 289434 'BioPreDyn:

83 New Bioinformatics Methods and Tools for Data-Driven Predictive Dynamic Modelling in 84 Biotechnological Applications". PM acknowledges the support of INRA. 
Table 1: Typical parameter values, derived from Nishio et al..

\begin{tabular}{ccc} 
parameter & value & units \\
\hline [gene] & $2.43 \times 10^{-7}$ & $\mathrm{mM}$ \\
[mRNA] & $10^{-4}$ & $\mathrm{mM}$ \\
[protein] & $10^{-2}$ & $\mathrm{mM}$ \\
[regulator:metabolite] & $10^{-4}$ & $\mathrm{mM}$ \\
transcription & 1 & $\mathrm{~s}^{-1}$ \\
translation & 0.183 & $\mathrm{~s}^{-1}$ \\
mRNA degradation & $2.43 \times 10^{-3}$ & $\mathrm{~s}^{-1}$ \\
protein degradation & $1.83 \times 10^{-3}$ & $\mathrm{~s}^{-1}$ \\
effector:site binding & $10^{5}$ & $\mathrm{mM}^{-1}$ \\
\hline
\end{tabular}

Table 2: Transcriptional regulatory interactions included in the model. Those in the top half are taken from Nishio et al..

\begin{tabular}{ccc} 
regulator & gene target & effect \\
\hline Mlc & $p t s G$ & - \\
& $p t s H$ & - \\
& $p t s I$ & - \\
Crp:cAMP & $c r p$ & + \\
& $c y a A$ & - \\
& $m l c$ & $+/-$ \\
& $p t s G$ & + \\
& $p t s H$ & + \\
& $p t s I$ & + \\
\hline Crp:cAMP & $p d h$ & - \\
Cra & $p f k$ & - \\
& $p p c$ & - \\
& $p t s H$ & - \\
& $p y k$ & - \\
& $t p i$ & - \\
PdhR:PYR & $z w f$ & - \\
& $p d h$ & - \\
\hline
\end{tabular}


Figure 1: A sudden decrease of glucose levels (from $2 \mathrm{mM}$ to $10 \mu \mathrm{M}$ ) is applied to the model at $\mathrm{t}=100 \mathrm{~s}$. The glucose uptake flux immediately decreases after the decrease of glucose concentration (A). This results in an increase of cAMP production (B) and of its concentration (C). In turn, the concentration of Crp:cAMP complex increases (D) and regulates the transcription of genes encoding metabolic enzymes (e.g. cyaA, E) and global regulators (e.g. crp, G). The dynamics of mRNA levels (e.g. cyaA, F, and crp, H) are slower.
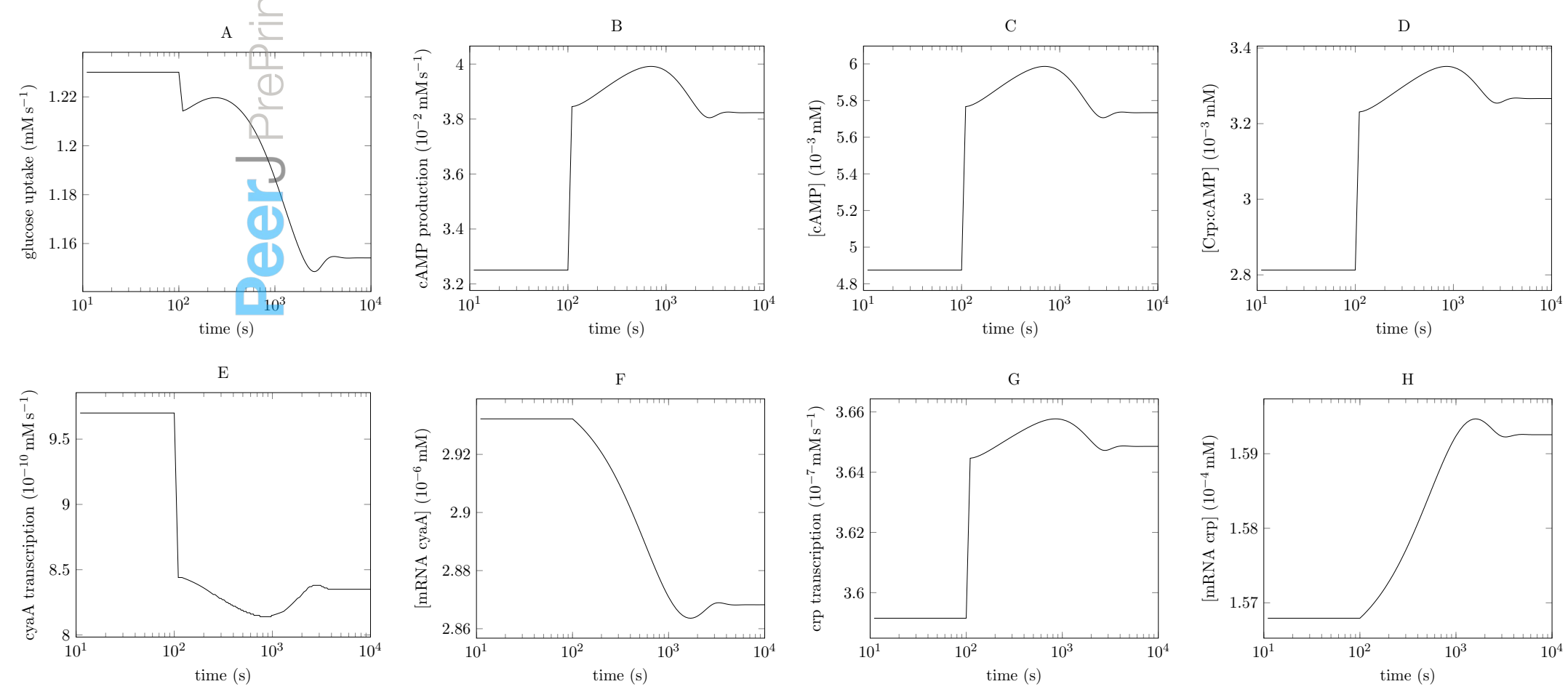
Figure 2: Steady-state response of the glucose uptake rate (solid, $\mathrm{mM} \mathrm{s}^{-1}$ ) and growth rate (dashed, $\mathrm{s}^{-1}$ ) (A), and of the partition of carbon at the glycolytic (solid) - PPP (dashed) node (B) when glucose concentration is varied between $10 \mu \mathrm{M}$ and $10 \mathrm{mM}$.

A

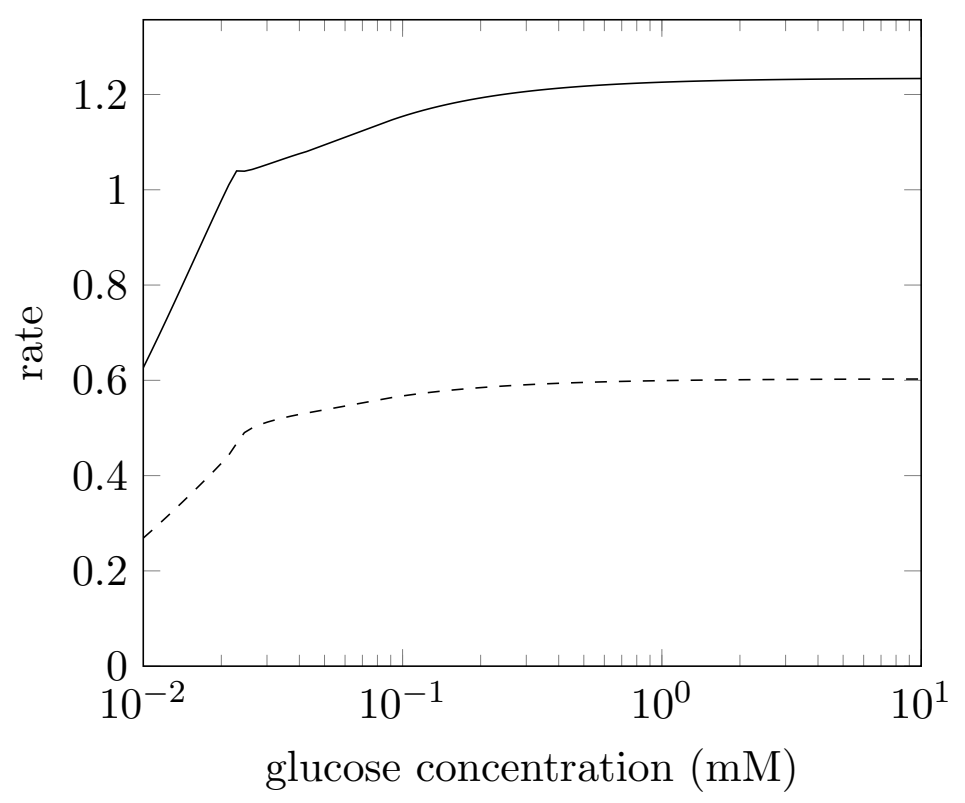

$\mathrm{B}$

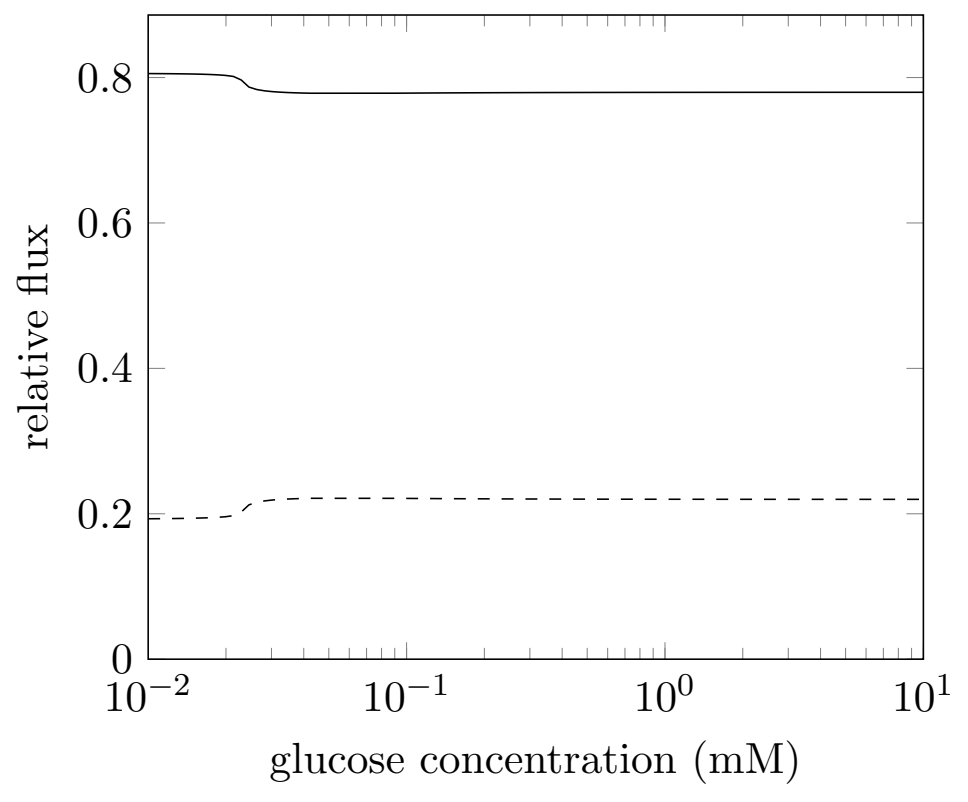




\section{References}

[1] Chassagnole C, Noisommit-Rizzi N, Schmid JW, Mauch K, Reuss M (2002) Dynamic modeling of the central carbon metabolism of Escherichia coli. Biotechnol Bioeng 79:53-73. doi:10.1002/bit.10288

[2] Kadir TA, Mannan AA, Kierzek AM, McFadden J, Shimizu K (2010) Modeling and simulation of the main metabolism in Escherichia coli and its several singlegene knockout mutants with experimental verification. Microb Cell Fact 9:88. doi:10.1186/1475-2859-9-88

[3] Kotte O, Zaugg JB, Heinemann M (2010) Bacterial adaptation through distributed sensing of metabolic fluxes. Mol Syst Biol 6:355. doi:10.1038/msb.2010.10

[4] Peskov K, Mogilevskaya E, Demin O (2012) Kinetic modelling of central carbon metabolism in Escherichia coli. FEBS $J$ 279:3374-3385. doi:10.1111/j.17424658.2012.08719.x

[5] Nishio Y, Usuda Y, Matsui K, Kurata H (2008) Computer-aided rational design of the phosphotransferase system for enhanced glucose uptake in Escherichia coli. Mol Syst Biol 4:160. doi:10.1038/msb4100201

[6] Li C, Donizelli M, Rodriguez N, Dharuri H, Endler L, Chelliah V, Li L, He E, Henry A, Stefan MI, Snoep JL, Hucka M, Le Novère N, Laibe C (2010) BioModels Database: An enhanced, curated and annotated resource for published quantitative kinetic models. BMC Syst Biol 4:92. doi:10.1186/1752-0509-4-92

[7] Hucka M, Finney A, Sauro H, Bolouri H, Doyle J, Kitano H, Arkin A, Bornstein B, Bray D, Cornish-Bowden A, Cuellar A, Dronov S, Gilles E, Ginkel M, Gor V, Goryanin I, Hedley W, Hodgman T, Hofmeyr J,Hunter P, Juty N, Kasberger J, Kremling A, Kummer U, Le Novère N, Loew L, Lucio D, Mendes P, Minch E, Mjolsness E, Nakayama Y, Nelson M, Nielsen P, Sakurada T, Schaff J, Shapiro B, Shimizu T, Spence H, Stelling J, Takahashi K, Tomita M, Wagner J, Wang J (2003) The systems biology markup language (SBML): a medium for representation and exchange of biochemical network models. Bioinformatics 19:524-531. doi:10.1093/bioinformatics/btg015

[8] Millard P, Massou S, Wittmann C, Portais JC, Létisse F (2014) Sampling of intracellular metabolites for stationary and non-stationary ${ }^{13} \mathbf{C}$ metabolic flux analysis in Escherichia coli. Anal Biochem 465C:38-49. doi:10.1016/j.ab.2014.07.026

[9] Smallbone K, Mendes P (2013) Large-scale metabolic models: from reconstruction to differential equations. Ind Biotech 9: 179-184. doi:10.1089/ind.2013.0003

[10] Stanford NJ, Lubitz T, Smallbone K, Klipp E, Mendes P, Liebermeister W (2013) Systematic construction of kinetic models from genome-scale metabolic networks. PLoS One 8:e79195. doi:10.1371/journal.pone.0079195

[11] Nanchen A, Schicker A, Sauer U (2006) Nonlinear dependency of intracellular fluxes on growth rate in miniaturized continuous cultures of Escherichia coli. App Env Microbiol 72(2):1164-1172. doi:10.1128/AEM.72.2.11641172.2006 